\title{
Multi-line treatment with tyrosine kinase inhibitors enabled in 4-year survival for patient with stage IV lung adenocarcinoma: a case report
}

\author{
Yan Yin, Jianwen Qin \\ Respiratory and Critical Care Medicine, Tianjin Chest Hospital, Tianjin, China \\ Correspondence to: Jianwen Qin. Respiratory and Critical Care Medicine, Tianjin Chest Hospital, No. 261 Taierzhuang South Road, Jinnan District, \\ Tianjin 300222, China. Email: qinjianwenyinyan@163.com.
}

\begin{abstract}
The tyrosine kinase inhibitors (TKIs) dramatically improve the clinical outcomes of non-small cell lung cancer (NSCLC) patients. Nevertheless, acquired resistance is practically inescapable in many patients. We recommended a case of lung adenocarcinoma patient, a 59-year-old Chinese woman, who was admitted to hospital with dyspnea after activity for no apparent reason. Computed tomography (CT) scan showed nodules in the anterior segment of the upper lobe of the right lung. This report presented the clinical characteristics, imaging findings, gene mutations, therapeutic regimen and outcome. The patient underwent two biopsies, found both EGFR 19 exon deletion and MET amplification, and EGFR T790M mutation was negative. In addition, $A L K$ was positive according to the Ventana IHC test. She received successively treatment of different EGFR-TKIs and ALK-TKI, namely gefitinib, osimertinib and crizotinib. Although EGFR T790M mutation was negative after blood sample biopsy, but the possibility of tissue positive was not excluded, and the family members refused issue biopsy and chemotherapy, therefore osimertinib was taken as second-line therapy. Although gefitinib has the most lasting effect of 25 months before osimertinib and crizotinib, the disease progressed due to the emergence of acquired resistance. The patient acquired 4-year survival after treated with multi-line TKIs. As far as we know, this was the first reported case that advanced NSCLC patient had achieved such a long survival after multi-line TKIs treatment. Molecular detection and rebiopsy play important roles in the selection of therapeutic regimen for TKIs. The main take-away lesson is that multi-line TKIs treatment was an effective clinical approach for patients with advanced NSCLC.
\end{abstract}

Keywords: Non-small cell lung cancer (NSCLC); tyrosine kinase inhibitor (TKI); rebiopsy; molecular detection

Submitted Jul 30, 2020. Accepted for publication Sep 25, 2020.

doi: 10.21037/apm-20-1514

View this article at: http://dx.doi.org/10.21037/apm-20-1514

\section{Background}

The treatment of non-small cell lung cancer (NSCLC) has entered the era of precision targeted therapy (1). Targeted drugs with better effects and fewer side effects are gradually replacing traditional chemotherapy drugs as first-line drugs (2). Tyrosine kinase inhibitors (TKIs) are a class of compounds that inhibit the activity of tyrosine kinase (3), and can be used as competitive inhibitors of adenosine triphosphate (ATP) binding to tyrosine kinases, as well as analogs of tyrosine. TKIs mainly inhibit the growth and proliferation of tumor cells by inhibiting cell signal transduction, thus promoting cell apoptosis (4). Because of its remarkable effect and good safety, individualized molecular targeted therapy for driver genes has become the standard treatment for advanced NSCLC (5). In particular, the discovery of epidermal growth factor receptor TKIs (EGFR-TKIs) and anaplastic lymphoma kinase TKIs (ALKTKIs) is a milestone in the development of personalized treatment of NSCLC (6). EGFR-TKIs and ALK-TKIs significantly improved the survival rate of NSCLC patients with the above mutations.

Gefitinib, a reversible TKI, is the first generation EGFR- 
TKI. It made $E G F R$ mutation positive patients obtain about 10 months of median progression-free survival (PFS) (7). However, most patients eventually developed acquired resistance, the important molecular mechanism of which is primarily the T790M mutation in EGFR gene exon 20 (8). Osimertinib, the third generation EGFR-TKI, is a class of irreversible drug, which has an apparent effect on EGFR sensitive mutations and T790M resistant mutations (9). It is the first targeted drug approved for treating EGFR T790M positive NSCLC patients. It is commonly used in patients with first and second generation EGFR-TKIs resistance. Crizotinib is the first generation ALK-TKI, and its main targets include ALK, c-Met, and ROS-1 (10). It is the only Food and Drug Administration (FDA)-approved targeted drug targeting two different molecular targets, ROS1 and ALK. However, within about 1 year of crizotinib treatment, many patients will develop resistance, leading to disease progression. The mechanisms of resistance include secondary mutations in $A L K$ kinase region and/ or amplification of $A L K$ gene copy number, as well as merger or secondary mutation of other genes and pathway activation, such as $c-K I T$ amplification, EGFR mutation or phosphorylation, KRAS mutation, etc. For patients with $A L K$ fusion gene positive, the second and third-generation ALK inhibitors are appropriately for patients with drug resistance to crizotinib. In addition, combination with other TKIs or second-line chemotherapy can also be used as the treatment option after crizotinib resistance.

So far, only few studies have reported the application of multi-line TKIs in cancer patients. In the current report, we introduced a 59-year-old Chinese female with lung adenocarcinoma. Because of the emergence of acquired resistance, the patient was treated with different EGFRTKIs and ALK-TKI in succession. To the best of our knowledge, this is the first report on the application of multiple TKIs in the multi-line treatment of advanced NSCLC patients. We present the following article in accordance with the CARE reporting checklist (available at http://dx.doi.org/10.21037/apm-20-1514).

\section{Case presentation}

A 59-year-old Chinese female was admitted to Tianjin Chest Hospital for diagnosis and treatment on December 9,2014 . The patient was breathless after activity without obvious reason 10 days ago, and B-scan ultrasonography indicated a large amount of pleural effusion on the right side. The physical examination revealed she had clear mental reaction, no cyanosis in the mouth and lips, low breathing sound in the right lung, a heart rate of 90 beats/min, regular heart rhythm, strong heart sounds, and a soft abdomen. After admission, the bloody pleural effusion was drained by thoracic tube drainage. CEA concentration in pleural fluid was more than $1,000 \mathrm{ng} / \mathrm{mL}$. The pathological diagnosis of pleural fluid sediment was adenocarcinoma. The computed tomography (CT) showed nodules exited in the anterior segment of the right upper lobe (Figure 1A). The patient was considered to be peripheral lung cancer, and unfortunately with right hilar and mediastinal lymph node metastasis.

The biopsy of the lesion confirmed that the patient was stage IV $\left(\mathrm{T}_{4} \mathrm{~N}_{2} \mathrm{M}_{1 \mathrm{a}}\right)$ lung adenocarcinoma. The physical status (PS) score was 0 . In the first-line therapy, the patient was treated with pemetrexil $(0.9 \mathrm{~g})$ combined with cisplatin (100 mg) chemotherapy between January and March 2015. After 2 cycles, the therapeutic effective evaluation was SD (Figure 1B). Pseudomonas aeruginosa preparation was locally injected into the pleural cavity to promote pleural adhesion and reduce pleural effusion. Subsequently, gefitinib $250 \mathrm{mg} / \mathrm{qd}$ was started in April 2015. Figure 1C showed the treatment effect after two months of treatment with gefitinib. At the 19th months of treatment with gefitinib, the patient developed metastases (Figure 1D) and she received pemetrexed $(0.9 \mathrm{~g})$ plus cisplatin $(100 \mathrm{mg})$ chemotherapy for 2 cycles from November to December 2016, and the curative effect was PR (Figure 1E). The follow-up treatment strategy was to continue oral administration of gefitinib (250 mg/qd) until May 2017 (Figure 1F). The EGFR T790M mutation analysis relied on blood was negative. The family members temporarily refused to biopsy, but the possibility of positive results in tissue samples was not excluded. In addition, they refused chemotherapy, so she started second-line therapy with osimertinib $(80 \mathrm{mg} / \mathrm{qd})$ in May 2017 (Figure 2A). At 2 months, CT showed the curative effect was SD (Figure $2 B$ ). Osimertinib treatment continued until November 2017, when the patient developed enlargement of the right cervical lymph node and distant lymph node metastasis (Figure 2C). The second biopsy was performed and the biopsy sample was sequenced by targeted next-generation sequencing (NGS) of the 12 lung cancer gene panel (ALK, ALK Fusion, BRAF, EGFR, ERBB2, FGFR1, MET, NRAS, KRAS, PIK3CA, RET Fusion, ROS1 Fusion and TP53). The results of sequencing showed EGFR exon 19 deletion in the form of p.Glu746_Ala750del (frequency $=13.55 \%$ ) and $M E T$ amplification (copy number $=5.22$ ). Furthermore, the ALK was positive according to 

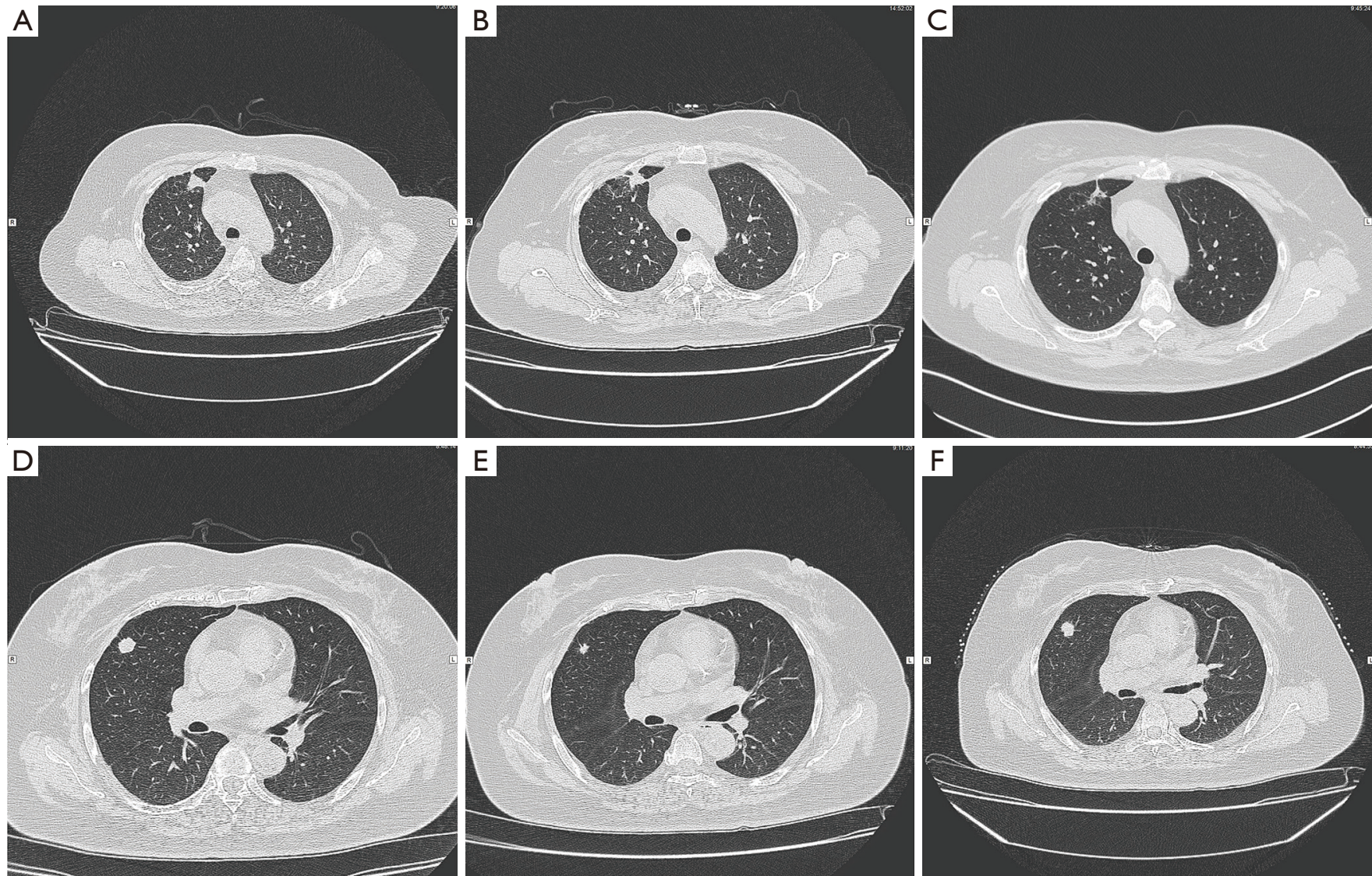

Figure 1 CT scan images demonstrated the effect of first-line treatment. (A) Before treatment initiation. (B) After treatment with 2 cycles of chemotherapy. (C) Gefitinib treatment for 2 months. (D) After 19 months of treatment with gefitinib. (E) Sequential chemotherapy for 2 cycles. (F) Treated with gefitinib until May 2017.
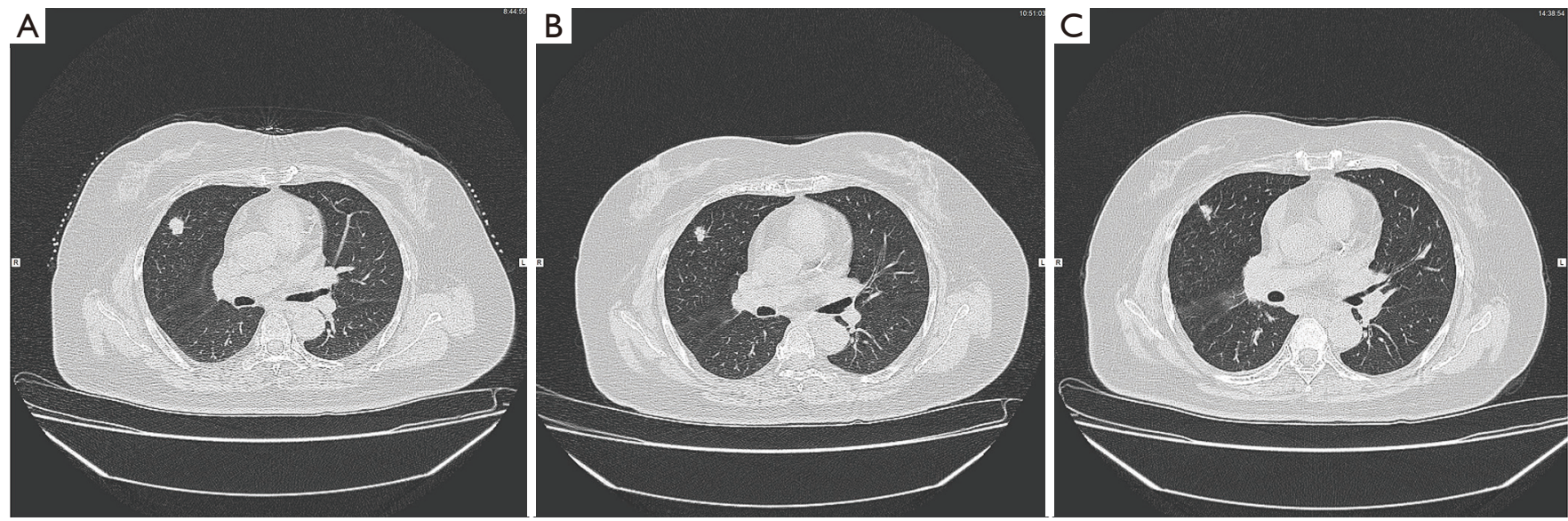

Figure 2 CT scan images demonstrated the effect of second-line therapy. (A) Before treated with osimertinib. (B) At 2 months of treatment with osimertinib. (C) Osimertinib treatment for 6 months. 

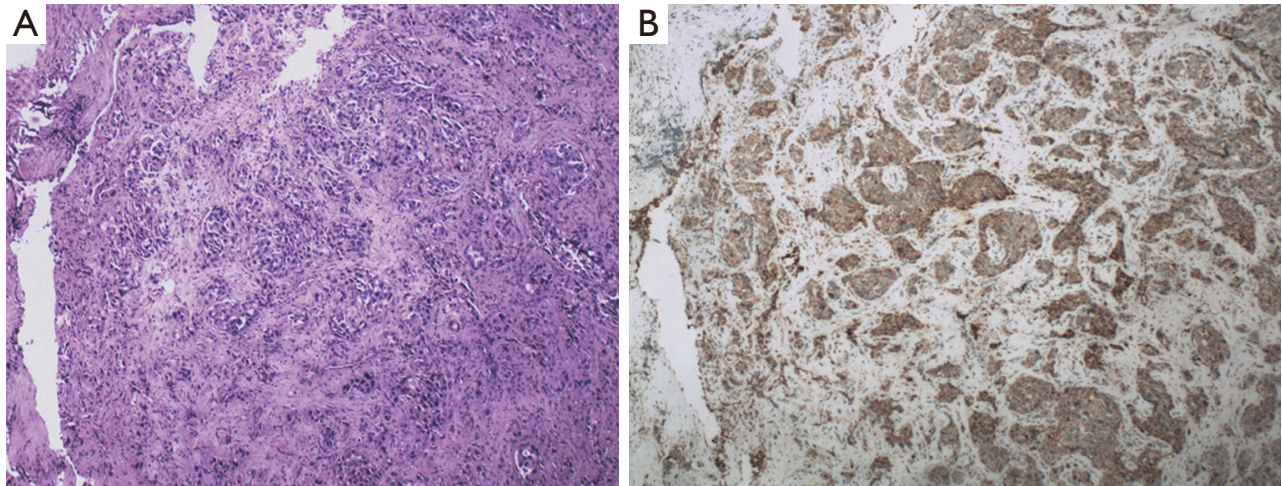

Figure 3 Expression of ALK in tumor tissues. (A) HE staining of tumor tissue samples. (B) Ventana IHC of ALK. Magnification: 100x. IHC, immunohistochemistry; ALK, anaplastic lymphoma kinase.
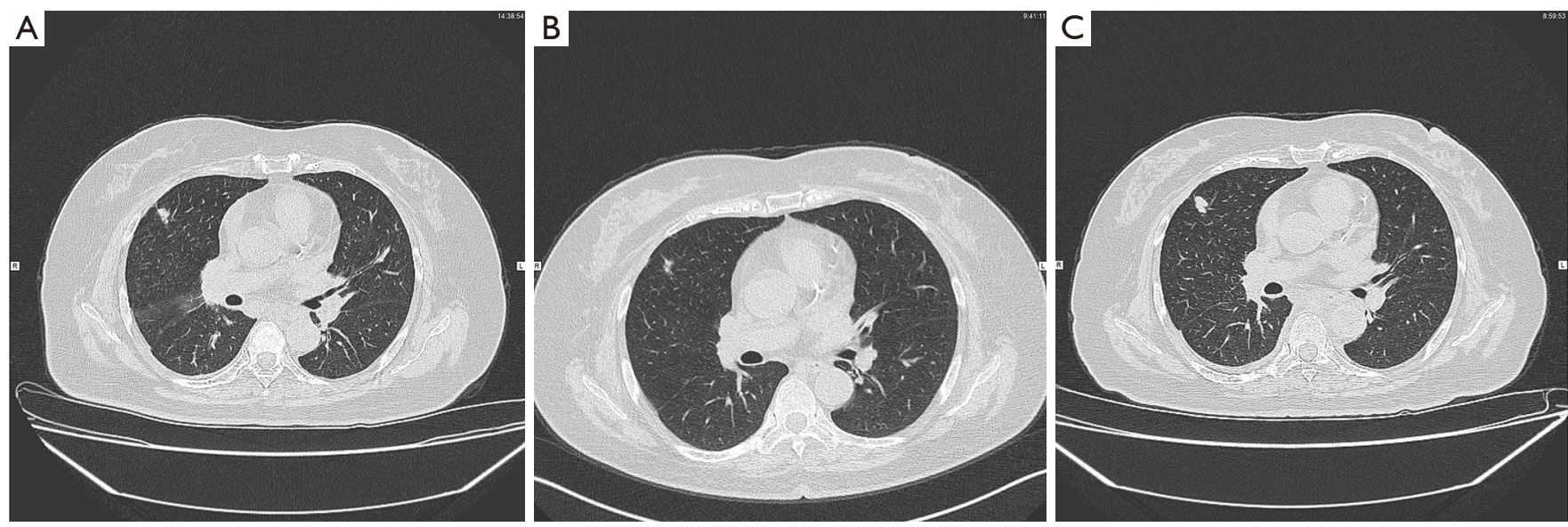

Figure 4 CT scan images demonstrated the effect of third-line therapy. (A) Before treated with crizotinib. (B) At 1 months of treatment with crizotinib. (C) Crizotinib treatment for 3 months.

the Ventana IHC (immunohistochemistry) assay (Figure 3). Therefore, the patient began to try third-line therapy with crizotinib (250 mg/bid) in December 2017 (Figure 4A). One month later, CT suggested PR (Figure 4B). However, the efficacy was evaluated as progressive disease (PD) after 3 months (Figure $4 C$ ). The next treatment strategy was the combination of crizotinib $(250 \mathrm{mg} / \mathrm{bid})$ and gefitinib $(250 \mathrm{mg} / \mathrm{qd})$ and also had certain curative effect. By the end of follow-up, the patient developed pulmonary embolism and lower limb venous thrombosis in August 2018 and died at the end of 2018. The course of treatment is presented in Figure 5. All procedures performed in studies involving human participants were in accordance with the ethical standards of the institutional and/or national research committee(s) and with the Declaration of Helsinki (as revised in 2013). The study was approved by the Ethical Committee of Tianjin Chest Hospital (No. 2020LW-009). Written informed consent for publication of the clinical information was obtained from the patient when she was admitted to the hospital.

\section{Discussion}

Traditional tumor treatment such as surgery, chemotherapy and radiotherapy often fail to achieve higher response rate and survival time in advanced NSCLC, and patients usually have poor prognosis. In recent years, multiple specific target sites have been discovered for treatment, so targeted therapy has become a research focus of advanced NSCLC (5). Among them, the treatment with TKIs has become an 


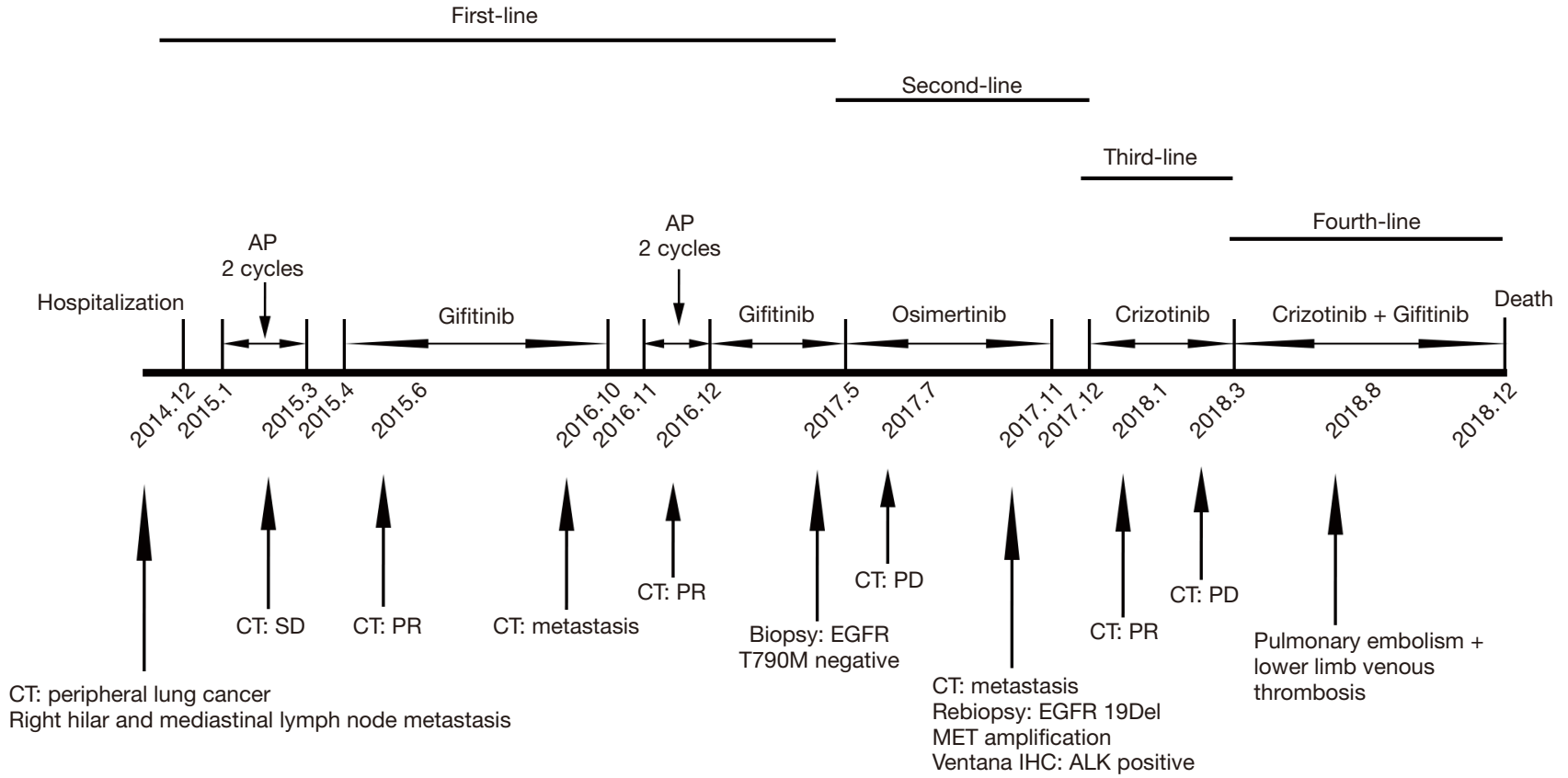

Figure 5 Multi-line TKIs therapy schedule. AP, pemetrexil combined with cisplatin chemotherapy; SD, stable disease; PR, partial response; $\mathrm{PD}$, progressive disease; TKIs, tyrosine kinase inhibitors.

indispensable method, opening the new way for the treatment of advanced NSCLC (11). Many kinds of TKIs, such as gefitinib (12), osimertinib (9), or crizotinib (13), have been used to treat several cases of NSCLC, but there were few cases in which the three drugs have been successively used for treatment as in this study. Although the 1-year and 5-year survival rate for patients with stage IV NSCLC is $15-35 \%$ and $2 \%$, respectively, the 59 -yearold stage IV lung adenocarcinoma patient achieved 4-year survival after multi-line TKIs treatment in present study. This is, to the best of our knowledge, the first reported case that stage IV NSCLC patient had achieved such a long survival after multi-line TKIs treatment. This showed that multi-line TKIs therapy was an effective method to improve the survival of patients with advanced NSCLC.

Among various TKIs, EGFR-TKIs are the most representative and has achieved marvelous results in the treatment of advanced NSCLC (14). Gefitinib is a selective EGFR-TKI that inhibits tumor growth, metastasis, and angiogenesis, and increases tumor cell apoptosis. However, even if the initial treatment of gefitinib is very effective, the disease will progress after a period of treatment (15). The cause of disease progression was often due to the patient appeared acquired resistance. Multiple mechanisms are currently known to cause acquired resistance to gefitinib in
NSCLC. The uppermost is a secondary EGFR mutation, T790M that renders gefitinib ineffective inhibitor of EGFR kinase activity (16). Almost 2/3 of patients with acquired resistance to gefitinib had secondary T790M mutations. In this report, it was exciting to note that the patient received effective treatment for 25 months after gefitinib treatment. However, the patient developed resistance after treated with gefitinib, but the T790M test was negative. This suggested that her resistance might be caused by other mechanisms, such as MET gene amplification, it can affect the PI3K/Akt pathway, which plays an important role in anti-apoptotic signaling. Besides, the test for ALK was negative at the outset, but after treatment with osimertinib, the ALK was positive. The underlying reasons might be that there was a large difference in the proportion of cancer cells in the two puncture samples before and after. Another reason might be that the antibodies and testing techniques used can also have an impact on test results.

During TKIs therapy, rebiopsy has become a new basis for treatment after disease progression, especially after the emergence of acquired drug resistance. Rebiopsy is highly recommended to identify the mechanism of acquired resistance to TKIs in advanced lung cancer (17). The rebiopsy is particularly important for the adjustment of the overall treatment strategy of the patients, which could 
accurately guide the clinical treatment of patients (18). Stoehlmacher et al. (19) underlined the significance of rebiopsies and opportunely molecular diagnostics to select suitable therapies after development of resistance to targeted therapies with EGFR-TKIs. It is suggested to analyze the dynamic changes of sensitive/resistant mutations or loss of driver genes in lung cancer based on rebiopsy or even multiple biopsies, so as to select the most appropriate individualized treatment for patients. In current article, gene detection based on the first biopsy sample revealed the presence of EGFR mutations, so gefitinib was chosen and has the most sustained effect of 25 months. After the patient developed osimertinib resistance, the second biopsy was performed, and MET gene amplification was detected by NGS. So crizotinib was applied to the third-line treatment, with a certain therapeutic effect.

Rebiopsy has been shown to significantly prolong the survival of patients in a number of studies (18). In this case, the patient was diagnosed with advanced NSCLC, after rebiopsy, she received multi-line TKI treatment, resulting in a survival time of 4 years that has never been reported. That is, through multiple biopsy, timely grasp the changes in gene status and perform multi-line TKI treatment can effectively improve the survival of patients with advanced NSCLC. But at the same time, there are certain limitations. Patients need to carry out multiple biopsies, which will cause physical pain to patients. Meanwhile, multiple tests require high costs, which increases the financial burden of patients. Moreover, rebiopsy may lead to serious complications. Therefore, this method is still far from clinical application, which requires not only the improvement of the method itself, but also the progress of high-throughput sequencing technology.

In conclusion, the emergence of TKIs has brought the treatment of NSCLC into the era of precision targeted therapy, and the survival of patients has clearly benefited. The molecular detection and rebiopsy play important roles in the selection of TKI treatment regimens. The mechanism of drug resistance of TKIs still require to be further explored.

\section{Acknowledgments}

Funding: None.

\section{Footnote}

Reporting Checklist: The authors have completed the CARE reporting checklist. Available at http://dx.doi.org/10.21037/ apm-20-1514

Conflicts of Interest: Both authors have completed the ICMJE uniform disclosure form (available at http://dx.doi. org/10.21037/apm-20-1514). The authors have no conflicts of interest to declare.

Etbical Statement: The authors are accountable for all aspects of the work in ensuring that questions related to the accuracy or integrity of any part of the work are appropriately investigated and resolved. All procedures performed in studies involving human participants were in accordance with the ethical standards of the institutional and/or national research committee(s) and with the Declaration of Helsinki (as revised in 2013). The study was approved by the Ethical Committee of Tianjin Chest Hospital (No. 2020LW-009). Written informed consent was obtained from the patient.

Open Access Statement: This is an Open Access article distributed in accordance with the Creative Commons Attribution-NonCommercial-NoDerivs 4.0 International License (CC BY-NC-ND 4.0), which permits the noncommercial replication and distribution of the article with the strict proviso that no changes or edits are made and the original work is properly cited (including links to both the formal publication through the relevant DOI and the license). See: https://creativecommons.org/licenses/by-nc-nd/4.0/.

\section{References}

1. Hirsch FR, Scagliotti GV, Mulshine JL, et al. Lung cancer: current therapies and new targeted treatments. Lancet 2017;389:299-311.

2. Solomon BJ, Mok T, Kim DW, et al. First-line crizotinib versus chemotherapy in ALK-positive lung cancer. N Engl J Med 2014;371:2167-77.

3. Krause DS, Van Etten RA. Tyrosine kinases as targets for cancer therapy. N Engl J Med 2005;353:172-87.

4. Arora A, Scholar EM. Role of tyrosine kinase inhibitors in cancer therapy. J Pharmacol Exp Ther 2005;315:971-9.

5. Kumarakulasinghe NB, van Zanwijk N, Soo RA. Molecular targeted therapy in the treatment of advanced stage non-small cell lung cancer (NSCLC). Respirology 2015;20:370-8.

6. Dahabreh IJ, Linardou H, Siannis F, et al. Somatic EGFR mutation and gene copy gain as predictive biomarkers for response to tyrosine kinase inhibitors in non-small cell 
lung cancer. Clin Cancer Res 2010;16:291-303.

7. Lin WC, Chiu CH, Liou JL, et al. Gefitinib as front-line treatment in Chinese patients with advanced non-smallcell lung cancer. Lung Cancer 2006;54:193-9.

8. Kosaka T, Yatabe Y, Endoh H, et al. Analysis of epidermal growth factor receptor gene mutation in patients with nonsmall cell lung cancer and acquired resistance to gefitinib. Clin Cancer Res 2006;12:5764-9.

9. Soria JC, Ohe Y, Vansteenkiste J, et al. Osimertinib in untreated EGFR-mutated advanced non-small-cell lung cancer. N Engl J Med 2018;378:113-25.

10. Heigener DF, Reck M. Crizotinib[M]//Small Molecules in Oncology. Springer, Cham, 2018:57-65.

11. Casaluce F, Sgambato A, Maione P, et al. ALK inhibitors: a new targeted therapy in the treatment of advanced NSCLC. Target Oncol 2013;8:55-67.

12. Sequist LV, Martins RG, Spigel D, et al. First-line gefitinib in patients with advanced non-small-cell lung cancer harboring somatic EGFR mutations. J Clin Oncol 2008;26:2442-9.

13. Drilon AE, Camidge DR, Ou SHI, et al. Efficacy and safety of crizotinib in patients (pts) with advanced MET exon 14-altered non-small cell lung cancer (NSCLC). J Clin Oncolo 2016;34:108.

Cite this article as: Yin Y, Qin J. Multi-line treatment with tyrosine kinase inhibitors enabled in 4-year survival for patient with stage IV lung adenocarcinoma: a case report. Ann Palliat Med 2021;10(6):6990-6996. doi: 10.21037/apm-20-1514
14. Mitsudomi T, Morita S, Yatabe Y, et al. West Japan Oncology Group Gefitinib versus cisplatin plus docetaxel in patients with non-small-cell lung cancer harbouring mutations of the epidermal growth factor receptor (WJTOG3405): an open label, randomised phase 3 trial. Lancet Oncol 2010;11:121-8.

15. Uramoto H, Sugio K, Oyama T, et al. Resistance to gefitinib. Int J Clin Oncol 2006;11:487-91.

16. Pao W, Miller VA, Politi KA, et al. Acquired resistance of lung adenocarcinomas to gefitinib or erlotinib is associated with a second mutation in the EGFR kinase domain. PLoS Med 2005;2:e73.

17. Hata A, Katakami N, Yoshioka H, et al. Rebiopsy of nonsmall cell lung cancer patients with acquired resistance to epidermal growth factor receptor-tyrosine kinase inhibitor: comparison between $\mathrm{t} 790 \mathrm{~m}$ mutation-positive and mutation-negative populations. Cancer 2013;119:4325-32.

18. Nosaki K, Satouchi M, Kurata T, et al. Re-biopsy status among non-small cell lung cancer patients in Japan: a retrospective study. Lung Cancer 2016;101:1-8.

19. Stoehlmacher-Williams J, Ehninger G, Zimmermann DR, et al. Targeting TKI-resistance in NSCLC: Importance of rebiopsy and molecular diagnostics-A case study. Cancer Treat Commun 2013;1:1-5. 